\title{
Índice de qualidade da dieta de mulheres usuárias de um programa de atividade física regular "Academia da Cidade", Aracajú, SE
}

\section{Healthy eating index of women exercising \\ in the "City Gym Program", Aracajú, Sergipe, Brazil}

Dayanne da COSTA2 Bruna Zavarize REIS²

Diva Aliete dos Santos VIEIRA ${ }^{2}$ Jamille Oliveira COSTA ${ }^{2}$

Pryscila Dryele Souza TEIXEIRA ${ }^{2}$

Oscar Felipe Falcão RAPOSO ${ }^{3}$

Flávia Emília Leite de LIMA ${ }^{4}$

Raquel Simões MENDES-NETTO ${ }^{4}$

RE S U M O

\section{Objetivo}

Avaliar a qualidade da dieta de mulheres praticantes de atividades físicas do Programa Academia da Cidade segundo idade e estado nutricional, Aracajú, Sergipe.

\section{Métodos}

Foram coletados dados sociodemográficos, antropométricos e dietéticos de 169 mulheres. O Índice de Qualidade da Dieta foi obtido a partir da média de aplicação de dois recordatórios de 24 horas. Os dados do Índice de Qualidade da Dieta total e de seus componentes foram analisados de acordo com o índice de massa corporal (obesos e não obesos) e a faixa etária (adultos jovens e adultos velhos). Foram realizadas análise estatística

1 Apoio: Fundação de Apoio a Pesquisa e Inovação Tecnológica do Estado de Sergipe. (Processo no 019.203.01119/2008-3).

2 Universidade Federal de Sergipe, Centro de Ciências Biológicas e da Saúde, Núcleo de Nutrição. São Cristovão, SE, Brasil.

3 Universidade Federal de Sergipe, Centro de Ciências Exatas e Tecnologia, Departamento de Estatística. São Cristovão, SE, Brasil.

${ }^{4}$ Universidade Federal de Sergipe, Centro de Ciências Biológicas e da Saúde, Núcleo de Nutrição. Av. Marechal Rondon, s/n., Jd Rosa Elze, Cidade Universitária Professor José Aloísio de Campos, São Cristovão, SE, Brasil. Correspondência para/Correspondence to: RS MENDES-NETTO. E-mail: <raquelufs@gmail.com>. 
732 | D COSTA et al.

descritiva e Análise de Variância para a comparação das médias de pontuação do Índice de Qualidade da Dieta entre os grupos.

\section{Resultados}

Na população estudada, com idade média de 49,2 anos, 24,85\% das mulheres foram consideradas obesas, e 43,20\% com obesidade abdominal. A pontuação média do Índice de Qualidade da Dieta foi 66,64 pontos, com 90,60\% da população apresentando dieta com necessidade de modificações. No componente hortaliças, as obesas apresentaram menor consumo do que as não obesas. Com relação à idade, aos componentes hortaliças, leite e derivados, à variedade da dieta e à pontuação final, obtiveram-se notas maiores entre adultos velhos.

\section{Conclusão}

A população apresentou hábitos alimentares inadequados principalmente entre as mais novas e as com excesso de peso. Assim, frisa-se a importância de estratégias educacionais voltadas à nutrição na efetivação de uma vida saudável.

Termos de indexação: Avaliação nutricional. Consumo de alimentos. Hábitos alimentares. Nutrição.

\section{A B S T R A C T}

\section{Objective}

The aim of the present study was to assess the diet quality of women exercising in the "City Gym Program" according to age and nutritional status, in Aracajú, Sergipe, Brazil.

\section{Methods}

Sociodemographic, anthropometric and dietary data of 169 women were collected. The Diet Quality Index was calculated by taking the average of two 24-hour recalls. Total Diet Quality Index data and its components were analyzed according to BMI (obese and non-obese) and age (young adults and older adults). Descriptive statistical analysis and analysis of variance were done for comparing the mean Diet Quality Index score of the groups.

\section{Results}

The average age of the sample of 169 women was 49.24 years, of which $24.85 \%$ were obese and $43.20 \%$ had abdominal obesity. The average Diet Quality Index score was 66.64 points, with $90.60 \%$ of the population requiring dietary changes. Obese women consumed fewer vegetables than non-obese. Meanwhile, older adults had better scores regarding vegetable intake, dairy product intake and diet variety, and a better final score.

\section{Conclusion}

The population presented poor eating habits, especially young and overweight women. Therefore, educational nutrition strategies are very important and highly recommended for the promotion of healthier lifestyles.

Indexing terms: Nutritional assessment. Food consumption. Food habits. Nutrition.

\section{N T R O D U ÇÃ O}

Nos últimos anos, o Brasil vem passando por um período de transição nutricional, caracterizado pela queda dos índices de desnutrição e aumento das taxas de sobrepeso e obesidade: esse quadro reflete a influência da industrialização e a importação de hábitos alimentares ocidentais, caracterizados pelo alto consumo de alimentos processados, de baixo teor nutricional e alto valor energético, em detrimento da ingestão de frutas, verduras, legumes e cereais integrais ${ }^{1}$.

No intuito de avaliar qualitativamente as dietas, tem-se procurado desenvolver instrumentos úteis e válidos que, ao mesmo tempo, permitam relacionar o consumo alimentar com a incidência de morbimortalidade². No entanto, ainda há publicações que avaliam os nutrientes de forma isolada, desconsiderando assim a análise global da dieta, o que, segundo Hann et 
al. ${ }^{3}$, não traduz a natureza multifatorial da dieta humana.

O Índice de Qualidade da Dieta (IQD) é um método de análise da alimentação de indivíduos, com a finalidade de avaliar a qualidade da dieta através de alguns componentes, como a avaliação da adequação dos grupos de alimentos, gordura total e saturada, colesterol, sódio e variedade da dieta$^{4}$. O IQD, proposto por Kennedy et al. ${ }^{4}$, foi considerado pela American Dietetic Association como um instrumento adequado para medir a qualidade global da alimentação na população, sendo capaz de refletir um gradiente de risco para muitas doenças crônicas relacionadas à alimentação, sendo validado por marcadores bioquímicos $^{5}$.

No Brasil, um estudo pioneiro avaliou o IQD de uma amostra representativa da população de quatro municípios do estado de São Paulo e demonstrou que apenas $4 \%$ da população tinham uma alimentação saudável ${ }^{6}$. Outro estudo também realizado no Brasil por Mota et al. ${ }^{7}$ mostrou que pontuações mais altas do índice de alimentação saudável associam-se de forma significativa à variedade da dieta, ao consumo aumentado de frutas, à adequação na ingestão de fibra dietética, ao baixo consumo de gordura total e saturada e às concentrações elevadas de alguns carotenóides e vitamina $C$. Outros estudos também compararam o IQD em função do estado nutricional e deposição de gordura abdominal, demonstraram que ser obeso aumenta a probabilidade de ter um IQD menor 8,9 .

Assim, as informações sobre a qualidade da dieta têm recebido atenção crescente, principalmente aquelas com elevado teor de lipídeos, energia e carboidratos simples, em detrimento do consumo de frutas, hortaliças e grãos integrais. Snyder et al. ${ }^{10}$ descrevem a importância da avaliação da qualidade da dieta de mulheres adultas, e associam o elevado consumo de gordura saturada, colesterol e açúcar simples ao risco de desenvolvimento de doenças crônicas não transmissíveis, como a obesidade e alguns tipos de câncer, como o de mama entre mulheres.
Combinar estratégias que visem a alterações, ao mesmo tempo, dos hábitos alimentares e dos níveis de atividade física parece ser fundamental na medida em que ambos são resultados da crescente industrialização e da movimentação para os centros urbanos de grandes parcelas da população. Pensando nisso, algumas cidades do Brasil implementaram programas de atividade física regular que são incentivados pelos governos municipais e estaduais, como, por exemplo, o Programa Academia da Cidade (PAC), em Aracajú (SE), em Recife (PE) e em Belo Horizonte (MG), cujo objetivo é promover saúde e contribuir para melhoria da qualidade de vida da população.

O programa oferece atividades físicas suficientes para que seus usuários se aproximem ou atinjam as recomendações mínimas da prática regular de atividade física sugerida para a manutenção de bons níveis de saúde ${ }^{11}$.

Considerando que esses indivíduos representam uma parcela da população com hábitos regulares de atividade física e que buscam melhores condições de saúde, é fundamental conhecer o estado nutricional e os hábitos alimentares dessa população, assim como o potencial risco de desenvolvimento de complicações metabólicas. Deve-se ressaltar, entretanto, que nenhum trabalho, até o momento, investigou a qualidade da alimentação desse grupo, sendo de relevante importância essa informação para futuras intervenções alimentares.

O presente trabalho teve como objetivo avaliar, segundo idade e estado nutricional, a qualidade da dieta de mulheres praticantes de atividades físicas do Programa Academia da Cidade, do município de Aracajú (SE).

\section{MÉ TODOS}

Trata-se de um estudo transversal, conduzido com usuárias adultas na faixa etária entre 20 anos e 59 anos e 11 meses do Programa Academia da Cidade de Aracaju (SE). O PAC oferece atividades físicas suficientes para que seus 
usuários se aproximem ou atinjam as recomendações mínimas da prática regular de atividade física sugerida para a manutenção de bons níveis de saúde. O PAC oferece atividades físicas que são realizadas em três dias da semana em cada um dos 15 polos. Cada aula tem a duração média de 60 minutos, quando são propostas atividades de avaliação física, aferição de pressão arterial, orientação e prescrição de caminhadas, alongamentos, aulas de ginástica (aeróbica e localizada), dança e yoga, além de atividades recreativas ${ }^{11}$.

Para cálculo do tamanho da amostra, utilizou-se um banco de dados fornecido pela coordenação do PAC, no qual havia informações sobre o número de usuários que participavam regularmente das aulas semanais durante os meses de outubro, novembro e dezembro de 2007 ( $n=603$ ). Como interessava para o estudo apenas indivíduos com idade entre 20 e 59 anos, foram eliminados do universo aqueles que se encontravam fora dessa faixa etária. A população total do estudo foi de 388 indivíduos, distribuídos em 15 polos do PAC.

O tamanho da amostra foi estimado considerando a prevalência de $40 \%$ para excesso de peso (Índice de Massa Corporal - IMC $>25 \mathrm{~kg} / \mathrm{m}^{2}$ ), estimativa obtida a partir dos dados cadastrais dessa população. Utilizando um erro aceitável de 2 pontos percentuais e um nível de $95 \%$ de confiança e acrescentando 15\% para suprir possíveis perdas e recusas, calculou-se como necessário estudar 180 indivíduos.

Os polos do Programa Academia da Cidade 13 de Julho, Castelo Branco, Inácio Barbosa e Bugio não foram considerados na amostra, uma vez que apresentaram baixa frequência de indivíduos inscritos no programa $(n<6)$ na ocasião em que a lista de alunos foi entregue pela coordenação. Diante disso, o $\mathrm{n}$ amostral necessário foi redistribuído nos outros polos $(n=11)$. O plano amostral até a escolha do indivíduo a ser entrevistado foi elaborado com base em técnicas de processos probabilísticos, por meio de amostra estratificada proporcional, em etapas.
Inicialmente, considerou-se cada polo como estrato a fim de garantir que, de forma irrestrita, representantes de toda a população do PAC fossem entrevistados. Para garantir a representatividade da população no estudo, foram selecionados indivíduos inscritos em todos os polos do PAC do município de Aracajú. Para tanto, após a divisão do $\mathrm{n}$ amostral por polo, procedeu-se à distribuição proporcional do número de indivíduos a serem entrevistados. Para a seleção, foi realizado um sorteio a partir de uma lista numerada, tendo sido definido que, prioritariamente, o sorteado seria o indivíduo a participar do estudo, e, na sua ausência, participaria o seguinte adulto da lista, entre 20 e 59 anos e presente no momento da coleta. Foram excluídos da amostra: gestantes, lactantes e pessoas com limitações para realização das medidas antropométricas.

Em dias pré-estabelecidos, os avaliadores conduziram a aplicação do questionário sobre dados sociodemográficos, seguido do primeiro recordatório alimentar. Com intervalo de no mínimo uma semana e no máximo quinze dias, foram aferidas as medidas antropométricas e repetida a aplicação do recordatório alimentar. Esse procedimento ocorreu até que todos os indivíduos elegíveis do polo da academia fossem avaliados.

Na avaliação antropométrica, as mulheres foram pesadas e medidas segundo técnicas preconizadas pelo Lohman ${ }^{12}$. Para aferição da altura, foi utilizado um estadiômetro portátil ALTUREXATA (escala bilateral de 35 a $213 \mathrm{~cm}$, resolução de $0,1 \mathrm{~cm})$. O peso foi medido por uma balança digital da marca Líder, modelo P-150M, com capacidade de $150 \mathrm{~kg}$ e graduação de $100 \mathrm{~g}$. A medição foi realizada duas vezes com os indivíduos descalços e com a nuca, as nádegas e os calcanhares encostados à parede. O valor médio das duas medições foi usado nas análises.

A partir dos escores de Massa Corporal (MC) e da estatura (EST), foi calculado o índice de massa corporal (IMC), classificado de acordo com a World Health Organization $(\mathrm{WHO})^{13}$. 
Também foi aferido o perímetro da cintura com fita métrica Sanny (American Medical do Brasil Ltda.), com precisão de $1 \mathrm{~mm}$, no ponto médio entre o último arco costal e a crista ilíaca, segundo a técnica de Lohman ${ }^{12}$, para a verificação das dimensões corporais das participantes, servindo como indicador de gordura visceral.

Para avaliação do consumo habitual de alimentos e nutrientes, foram aplicados dois recordatórios alimentares, com intervalo de no mínimo uma semana e de no máximo 15 dias entre o primeiro e o segundo. A partir dos dados obtidos, os cálculos para quantificar o consumo de energia e de nutrientes foram realizados com o auxilio do software Virtual Nutri Plus, versão 2.0, e complementados com a Tabela de Composição de Alimentos $^{14}$. Vale ressaltar que, como o número de alimentos aumenta rapidamente no mercado, algumas informações que não existiam no programa ou nas tabelas utilizadas foram obtidas a partir dos rótulos dos produtos descritos pela participante. Assim também as preparações caseiras foram desmembradas em seus respectivos ingredientes e classificadas segundo os grupos da pirâmide alimentar.

Para avaliação da qualidade da dieta, foi utilizado o IQD, proposto por Kennedy et al. ${ }^{4} \mathrm{e}$ adaptado por Fisberg et al. ${ }^{2}$, para a população brasileira. Esse índice é obtido por uma pontuação distribuída em dez componentes que caracterizam diferentes aspectos de uma dieta saudável. Cada componente foi avaliado e pontuado de zero a dez, sendo os valores intermediários calculados proporcionalmente. O IQD, adaptado por Fisberg et al. ${ }^{2}$, alterou a gordura saturada pelo grupo de leguminosas devido ao hábito alimentar brasileiro de consumir feijão, e sua inclusão no grupo das carnes e ovos poderia levar a superestimação da ingestão desse grupo. Como o índice de alimentação saudável inclui em sua pontuação as porções alimentares estabelecidas para a população americana, optou-se por utilizar as recomendações de porções estabelecidas pelo Guia Alimentar para a População Brasileira.
Nesse índice, seis componentes são representados pelos grupos de alimentos (cereais, pães, tubérculos e raízes; hortaliças; frutas; leite e derivados; carnes e ovos; e leguminosas) e avaliados segundo o guia alimentar e a pirâmide alimentar adaptada de Philippi et al. ${ }^{15}$; três componentes são representados pelos nutrientes (gordura total; colesterol e sódio), e o último, pela variedade da dieta, e medido a partir dos diferentes tipos de alimentos consumidos no dia. Para o consumo inferior a três, o escore estabelecido era zero, e para um consumo superior a oito, a pontuação dada foi dez. É importante destacar que, se o mesmo alimento fosse consumido mudando apenas sua forma de preparação (frito, cozido etc.), ele seria computado apenas uma vez. Com base nos dois recordatórios, foi realizada a soma da pontuação de cada componente, que gerou o escore final do IQD.

O processamento de dados foi realizado com o auxílio de um programa "IQD-INDEX", que calcula e atribui a pontuação para cada indivíduo, tendo sido desenvolvido em linguagem Statistical Package for the Social Sciences (SPSS) (versão 8) para Windows, especificamente para este estu$\mathrm{do}^{16}$. O escore total dos indivíduos foi dividido em três categorias de acordo com Bowman et al. ${ }^{17}$ : a dieta é considerada inadequada quando sua pontuação é inferior a 51 pontos; entre 51 e 80 pontos, é uma dieta que necessita de modificação; e para pontuação maior que 81 pontos, a dieta é considerada saudável.

Para análise dos dados foram utilizadas estatísticas descritivas de frequências absolutas e relativas, média, desvio-padrão para a apresentação dos dados antropométricos, idade e pontuação do IQD.

Os indivíduos do estudo foram agrupados em dois grupos segundo o estado nutricional e a classificação do risco de excesso de gordura visceral. Para a comparação das médias de pontuação do IQD e dos seus componentes, os indivíduos do estudo foram classificados em grupos de acordo com o IMC - obesos (IMC $\left.\geq 30 \mathrm{~kg} / \mathrm{m}^{2}\right)$ e não obesos $\left(\mathrm{IMC}<30 \mathrm{~kg} / \mathrm{m}^{2}\right)$-, e de acordo com a 
circunferência da cintura - com risco para obesidade abdominal (circunferência da cintura $\geq 88 \mathrm{~cm}$ ) e sem risco para a obesidade abdominal (circunferência da cintura $<88 \mathrm{~cm})^{18}$.

Para a comparação das médias de pontuação do IQD dos grupos e dos componentes de IQD, foi utilizada a análise de múltiplas médias (Anova), sendo adotado como nível de significância 5\% $(p<0,05)$.

A digitação dos dados foi feita em planilha Excel ${ }^{\circledR}$ e posteriormente exportada e analisada com o auxílio do programa (SPSS ${ }^{\circledR}$ ) do Windows, versão 17.0 .

O estudo foi conduzido dentro dos padrões exigidos pela Declaração de Helsinki (2000), e aprovado pelo Comitê de Ética e Pesquisa do Hospital Universitário, Universidade Federal de Sergipe (UFS). (C.A.A.E. 0097.0.107.000-08). Todos os indivíduos envolvidos assinaram o Termo de Consentimento Livre e Esclarecido.

\section{RESULTADOS}

Participaram do estudo 182 mulheres, no entanto, devido à inconsistência de dados coletados de 13 indivíduos, a amostra final foi de 169 participantes de 11 polos do PAC. A idade média foi de 49,24 anos, IMC de 27,33 kg/m² e média da circunferência da cintura de $87,24 \mathrm{~cm}$ (Tabela 1). Nesse grupo, $24,85 \%$ eram obesas

Tabela 1. Média, desvio-padrão, mínimo e máximo de variáveis antropométricas e do Índice de Qualidade da Dieta entre mulheres do Programa Academia da Cidade. Aracajú (SE), 2009.

\begin{tabular}{|c|c|c|c|c|}
\hline Variáveis & Média & Desvio-Padrão & Mínimo & Máximo \\
\hline Idade (anos) & 49,24 & 9,11 & 20,00 & 59,00 \\
\hline Peso $(k g)$ & 65,60 & 12,29 & 36,60 & 100,50 \\
\hline Estatura (m) & 1,54 & 0,59 & 1,36 & 1,72 \\
\hline IMC (kg/m²) & 27,33 & 4,74 & 15,35 & 40,11 \\
\hline $\mathrm{CC}(\mathrm{cm})$ & 87,24 & 10,30 & 59,90 & 114,50 \\
\hline
\end{tabular}

IMC: Índice de Massa Corporal; CC: Circunferência da Cintura; IQD: Índice de Qualidade da Dieta.

Tabela 2. Média, desvio-padrão e prevalência dos escores dos componentes do Índice de Qualidade da Dieta e classificação da qualidade da dieta das mulheres do Programa Academia da Cidade. Aracajú (SE), 2009.

\begin{tabular}{|c|c|c|c|c|}
\hline Itens & Média & Desvio-Padrão & $\%$ de escore 0 & $\%$ de escore 10 \\
\hline Cereais, pães, raízes & 5,60 & 2,07 & 0,6 & 2,30 \\
\hline Hortaliças & 3,12 & 2,73 & 18,7 & 0,60 \\
\hline Frutas & 7,77 & 3,01 & 5,3 & 48,00 \\
\hline Leite e derivados & 2,93 & 2,20 & 11,1 & 0,00 \\
\hline Carnes e ovos & 6,03 & 3,47 & 12,3 & 27,50 \\
\hline Leguminosas & 6,00 & 3,06 & 7,0 & 7,00 \\
\hline Gordura Total & 8,82 & 1,83 & 0,0 & 46,20 \\
\hline Colesterol & 8,95 & 1,94 & 0,0 & 70,20 \\
\hline Sódio & 9,17 & 1,87 & 0,0 & 63,70 \\
\hline Variedade da dieta & 8,21 & 1,31 & 0,0 & 11,10 \\
\hline Pontuação do IQD & 66,64 & 9,30 & 0,0 & 0,00 \\
\hline \multicolumn{5}{|c|}{ Qualidade da Dieta - Classificação de Bowman } \\
\hline & $\mathrm{N}$ & \multicolumn{2}{|l|}{$\%$} \\
\hline \multicolumn{2}{|c|}{$<51$ "dieta pobre" } & 8 & \multicolumn{2}{|l|}{4,7} \\
\hline \multicolumn{2}{|c|}{51 - 80 "dieta que necessita de mudança" } & 155 & \multicolumn{2}{|l|}{90,6} \\
\hline \multicolumn{2}{|c|}{$>80$ "dieta saudável" } & 8 & \multicolumn{2}{|l|}{4,7} \\
\hline
\end{tabular}

IQD: Índice de Qualidade da Dieta. 
$\left(\mathrm{IMC}>30 \mathrm{Kg} / \mathrm{m}^{2}\right)$ e $43,20 \%$ apresentaram obesidade abdominal $(C C>88 \mathrm{~cm})$.

A Tabela 2 apresenta a média, o desvio-padrão e o percentual de escore 0 e 10 encontrado em cada componente analisado do IQD, como também no IQD total. A população estudada apresentou melhores médias para os componentes gordura total, colesterol, sódio e variedade da dieta, e os componentes colesterol e sódio apresentaram maiores percentuais para escore 10. A pontuação média do IQD foi de 66,64 pontos, com $90,6 \%$ da população apresentando dieta com necessidade de modificações.
Na Tabela 3, estão apresentados os escores de cada componente dietético analisado de acordo com a classificação do estado nutricional em obesos e não obesos. Nota-se que, apenas para o componente das hortaliças, houve diferença significativa entre obesos e não obesos, com o grupo de obesos apresentando menor consumo.

Na busca das diferenças quanto às escolhas alimentares no decorrer da vida e se estas são influenciadas pela idade, foram analisados a pontuação do IQD e de seus componentes. Separou-se a população em dois grupos: o de adultos mais velhos (41 a 59 anos e 11 meses) e o de

Tabela 3. Comparação das notas dos grupos e do IQD entre indivíduos não obesos e obesos, segundo o IMC. Programa Academia da Cidade. Aracajú (SE), 2009.

\begin{tabular}{|c|c|c|c|c|}
\hline \multirow{2}{*}{ Componentes do IQD } & \multicolumn{2}{|c|}{$\mathrm{IMC} \leq 30 \mathrm{~kg} / \mathrm{m}^{2}$} & \multicolumn{2}{|c|}{$\mathrm{IMC}>30 \mathrm{~kg} / \mathrm{m}^{2}$} \\
\hline & Média & Desvio-Padrão & Média & Desvio-Padrão \\
\hline Cereais, pães, raízes & 5,49 & 2,04 & 5,92 & 2,18 \\
\hline Hortaliças & $3,37^{*}$ & 2,84 & 2,32 & 2,15 \\
\hline Frutas & 7,83 & 2,96 & 7,55 & 3,24 \\
\hline Leite e derivados & 2,91 & 2,16 & 2,97 & 2,30 \\
\hline Carnes e ovos & 6,20 & 3,39 & 5,77 & 3,61 \\
\hline Leguminosas & 5,86 & 3,11 & 6,23 & 2,89 \\
\hline Gordura total & 8,85 & 2,07 & 8,91 & 1,77 \\
\hline Colesterol & 9,00 & 1,97 & 9,00 & 2,46 \\
\hline Sódio & 9,21 & 1,94 & 9,02 & 1,66 \\
\hline Variedade da dieta & 8,29 & 1,34 & 8,02 & 1,21 \\
\hline Pontuação do IQD & 66,96 & 9,92 & 65,52 & 7,32 \\
\hline
\end{tabular}

* $p<0,05$; IMC: Índice de Massa Corporal; IQD: Índice de Qualidade da Dieta.

Tabela 4. Comparação das notas dos grupos e do IQD entre indivíduos adultos jovens e adultos mais velhos. Programa Academia da Cidade. Aracajú (SE), 2009.

\begin{tabular}{|c|c|c|c|c|}
\hline \multirow{2}{*}{ Componentes do IQD } & \multicolumn{2}{|c|}{ Idade 20 - 40 anos } & \multicolumn{2}{|c|}{ Idade 41 - 59 anos } \\
\hline & Média & Desvio-Padrão & Média & Desvio-Padrão \\
\hline Cereais, pães, raízes & 5,81 & 2,28 & 5,56 & 2,05 \\
\hline Hortaliças & 2,16 & 2,41 & $3,29^{*}$ & 2,47 \\
\hline Frutas & 7,27 & 3,34 & 7,85 & 2,97 \\
\hline Leite e derivados & 1,96 & 1,74 & $3,11^{*}$ & 2,23 \\
\hline Carnes e ovos & 6,04 & 3,63 & 6,11 & 3,42 \\
\hline Leguminosas & 5,94 & 3,32 & 5,96 & 3,01 \\
\hline Gordura total & 8,80 & 1,82 & 8,88 & 2,03 \\
\hline Colesterol & 9,21 & 2,90 & 8,96 & 1,91 \\
\hline Sódio & 8,88 & 2,30 & 9,22 & 1,79 \\
\hline Variedade da dieta & 7,69 & 1,31 & $8,32^{*}$ & 1,29 \\
\hline Pontuação do IQD & 63,27 & 7,99 & $67,24^{*}$ & 9,47 \\
\hline
\end{tabular}

*p<0,05; IQD: Índice de Qualidade da Dieta. 
jovens adultos (20 a 40 anos). Assim, foi possível verificar que para os componentes, hortaliças, leite e derivados, variedade da dieta e pontuação final, os adultos mais velhos obtiveram notas significativamente maiores quando comparados aos adultos mais jovens (Tabela 4).

\section{I S C U S S Ã O}

Hábitos alimentares inadequados podem ser responsáveis pela alta prevalência de obesidade que vem sendo encontrada em diferentes populações $^{19}$, especialmente entre mulheres ${ }^{20}$. A avaliação da qualidade da dieta pelo IQD permite analisar a alimentação dos indivíduos, determinando a qualidade da dieta através de um ou mais parâmetros, como ingestão adequada de nutrientes, número de porções dos grupos alimentares e quantidade de diferentes alimentos presentes na dieta $^{21}$. Guo et al. ${ }^{8}$, ao estudar a relação entre o IQD e a obesidade, demonstraram uma associação significativamente positiva entre baixos níveis de adequação da dieta e sobrepeso e obesidade.

A população do presente estudo, além de ter apresentado um perfil nutricional caracterizado pela alta prevalência de obesidade, obteve um IQD médio de 66,64 pontos, com $90,6 \%$ das participantes apresentando uma dieta que necessita de modificações. Dados semelhantes foram encontrados em estudos realizados com a população americana ${ }^{8,10,22}$, canadense ${ }^{23}$ e brasileira ${ }^{7,16}$, tendo sido as mulheres que apresentaram pontuações mais baixas $7,8,23$. Nota-se que, em todos os estudos, as populações apresentaram dietas com necessidades de modificação, ou seja, destaca-se a necessidade de intervenções nos hábitos alimentares dessas populações a fim de se evitarem possíveis patologias no futuro.

Pôde-se observar também que 4,7\% da população estudada obtiveram pontuação inferior a 50 pontos, ou seja, "dieta pobre", dado também identificado em outro estudo brasileiro conduzido no município de São Paulo ${ }^{16}$. Esse fato é relevante e denota a grande importância da edu- cação nutricional voltada para esses grupos como forma de desenvolvimento de estratégias que possam modificar o padrão alimentar inadequado.

Comparando esse resultado com dados internacionais, percebe-se que essas porcentagens são ainda maiores. Segundo estudo realizado por Guo et al. ${ }^{8}, 17,8 \%$ da população obtiveram dieta pobre, e $15,8 \%$ das mulheres, pontuação menor que 51 pontos. Estudo realizado com a população de Mississippi, por McCabe-Sellers et al. ${ }^{24}$, também encontrou que $24,8 \%$ da população apresentaram dieta caracterizada como "pobre", e, quanto ao gênero, as mulheres apresentaram a porcentagem de $24,3 \%$. Assim, esses autores afirmam que a falta de conhecimento nutricional e, portanto, uma incapacidade de identificar alimentos nutritivos podem ser a razão dessas populações apresentarem menores pontuações do IQD.

Ao analisarem-se os componentes do IQD e o IQD total da população estudada, notam-se valores de média e prevalência de escore 10 baixos para os componentes hortaliças e leite e derivados. Um estudo realizado na cidade de Porto Alegre, que analisou o IQD de dois grupos - um grupo-controle e um grupo exposto a um programa de reeducação alimentar por 18 meses -, encontrou que o grupo-controle apresentou menores pontuações para os grupos de hortaliças, leite e derivados e frutas em relação ao grupo exposto, mostrando assim a importância da educação alimentar como ferramenta na promoção da alimentação saudável21.

Além da avaliação da qualidade da dieta de uma população, vários estudos têm buscado relacionar também o IQD total e seus componentes com fatores antropométricos, como o estado nutricional e a distribuição da gordura corporal, e fatores sociodemográficos, como idade e ren$d a^{16}$. No presente estudo, foi possível identificar que o excesso de peso estava relacionado com o menor consumo de hortaliças.

Guo et al. ${ }^{8}$, estudando 10930 indivíduos - dos quais $24,77 \%$ eram obesos -, através dos dados do NHANES III, obtiveram como resultado que os obesos possuíram escores no com- 
ponente hortaliças mais baixos se comparados aos não obesos. O menor consumo de hortaliças entre as obesas também foi encontrado no estudo de Nemby et al.25, que analisou 459 indivíduos que viviam em Baltimore.

Tande et al. ${ }^{9}$, no ano de 2009, analisando dados do NHANES III de 15658 pessoas, obtiveram dados significativos para o excesso de gordura abdominal, menores pontuações nos componentes leite e derivados, gordura total e variedade da dieta entre mulheres e menor pontuação nos componentes gordura total e saturada entre os homens.

As pontuações do IQD e de seus componentes também foram comparados entre dois grupos etários: adultos jovens (20 a 40 anos) e adultos mais velhos (41 a 59 anos). Foi possível observar que os indivíduos ditos mais velhos tiveram escores mais altos para pontuação total do IQD, hortaliças, leite e derivados e variedade da dieta. Dados semelhantes foram encontrados no estudo realizado por Guo et al. ${ }^{8}$, no qual as pessoas mais velhas também obtiveram pontuações mais altas no IQD médio e seus componentes. Alguns autores defendem que, com o avançar da idade, as pessoas passam a se preocupar mais com a saúde e buscam como medida terapêutica a prática de atividade física ${ }^{26}$ e a melhora da alimentação ${ }^{27}$.

Estudo realizado por Fernandes et al. ${ }^{28}$, na cidade de Porto Alegre, no ano de 2003, buscou conhecer os fatores que levavam as pessoas a utilizarem mais os serviços médicos. Dados desse estudo mostraram que o avanço da idade e a autopercepção de piora da saúde foram os fatores que mais levaram a maioria das mulheres a realizarem consultas médicas ou utilizarem as unidades de saúde da família.

Apesar de as pessoas ditas mais velhas obterem escores significativamente mais altos no componente de leite e de derivados em relação às mais jovens, no presente estudo, pôde-se perceber que não houve o escore máximo no componente leite e derivados para nenhum indivíduo da população. Dados semelhantes foram encon- trados por Freedman et al. ${ }^{29}$, que, estudando uma população de 738 mulheres norte-americanas, encontrou que $100 \%$ dessa população também não obteve o escore dez para esse componente.

O consumo inadequado de leite e derivados pode representar implicações importantes na saúde óssea de mulheres, visto que o comprometimento na ingestão total de cálcio favorece o desenvolvimento da osteoporose ${ }^{30}$.

\section{O N CLUS Ã O}

Os resultados obtidos evidenciam que a população apresentou hábitos alimentares inadequados, principalmente referentes ao consumo de hortaliças e de leite e derivados. Destaca-se, no entanto, a importância da realização de mais estudos que avaliem a influência do estado nutricional e da faixa etária na qualidade da dieta de mulheres adultas, e a importância de constantes estratégias educacionais voltadas à nutrição na efetivação de uma vida mais saudável para toda a população, principalmente as praticantes de atividade física regular, já que buscam no exercício físico benefícios para a obtenção de hábitos saudáveis, manutenção do peso corporal ideal e assim a prevenção de doenças crônicas associadas.

\section{AGRADECIMENTOS}

Aos alunos e aos professores do Programa Academia da Cidade, em nome do coordenador geral Braulio César de Alcântara Mendonça, pelo apoio na realização do projeto.

\section{COLABORADORES}

D COSTA participou na concepção do projeto, fazendo tanto coleta como realização da discussão dos resultados e redação do artigo. BZ REIS, DVS VIEIRA, FEL LIMA, JO COSTA e PDS TEIXEIRA participaram na concpção do projeto, coleta e discussão dos dados. RS MENDES-NETTO coordenou o projetivo e esteve envolvida em todas as etapas, desde a revisão crítica do 
conteúdo intelectual abordado como também a aprovação final do artigo finalizado. OFF RAPOSO realizou a análise do projeto desde a concepção do projeto (cáculo amostral até o processamento e análise dos dados).

\section{REFERÊ NCIAS}

1. Sichieri R. Dietary patterns and their associations with obesity in the brazilian city of Rio de Janeiro. Obes Res. 2002; 10(1):42-48.

2. Fisberg RM, Slater B, Barros RR, Lima FD, Cesar $C L G$, Carandina $L$, et al. Índice de qualidade da dieta: avaliação da adaptação e aplicabilidade. Rev Nutr. 2004; 17(3):301-08. doi: 10.1590/S1415-52 732004000300003

3. Hann CS, Rock CL, King I, Drewnowski A. Validation of healthy eating index with use of plasma biomarkers in a clinical sample of women. Am J Clin Nutr. 2001; 74(1):479-86.

4. Kennedy ET, Ohls J, Carlson S, Fleming K. The healthy eating index: design and applications. J Am Diet Assoc. 1995; 95(10):1103-09.

5. Weinstein SJ, Vogt TM, Gerrior SA. Healthy eating index scores are associated with blood nutrient concentrations in the Third Health and Nutrition Examination Survey. J Am Diet Assoc. 2004; 104(4): 576-84.

6. Morimoto JM, Latorre MRDO, César CLG, Carandina L, Barros MBA, Goldbaum M, et al. Fatores associados à qualidade da dieta de adultos residentes na Região Metropolitana de São Paulo, Brasil, 2002. Cad Saúde Pública. 2008; 24(1): 169-78.

7. Mota JF, Rinaldi AEM, Pereira AF, Maestá N, Scarpin MM, Burini RC. Adaptação do índice de alimentação saudável ao guia alimentar da população brasileira. Rev Nutr. 2008; 21(5):545-52. doi: 10.1590/S1415-52732008000500007.

8. Guo X, Warden BA, Paeratakul S, Bray GA. Healthy eating index and obesity. Eur J Clin Nutr. 2004; 58(12):1580-86

9. Tande DL, Magel R, Strand BN. Healthy eating index and abdominal obesity. Public Health Nutr. 2009; 13(2):208-14.

10. Snyder DC, Sloane R, Haines PS, Miller P, Clipp EC, Morey MC, et al. The diet quality index-revised: a tool to promote and evaluate dietary change amon older cancer survivors enrolled in a home-based intervention trial. J Am Diet Assoc. 2007; 107(1): 1519-29.
11. Mendonça BCA, Toscano JJO, Oliveira ACC. Programa Academia da Cidade Aracajú: promovendo saúde por meio da atividade física. Rev Bras Ativ Fís Saúde. 2009; 14(3): 211-16.

12. Lohman TG. Advances in body composition assessment. Champaign (IL): Human Kinetics Publishers; 1992.

13. World Health Organization. Obesity: preventing and managing the global epidemic. Geneva: WHO; 1998. Report of a WHO consultation on obesity.

14. Tabela Brasileira de Composição de Alimentos. Versão II. $2^{\circ}$ ed. Campinas: Unicamp; 2006.

15. Philippi ST, Latterza AR, Cruz ATR, Ribeiro LC. Pirâmide alimentar adaptada: guia para escolha dos alimentos. Rev Nutr. 1999; 12(1):65-8. doi: 10.1590/S1415-52731999000100006.

16. Fisberg RM, Morimoto JM, Slater B, Barros MBA, Carandina L, Goldbaum MJ, et al. Dietary quality and associated factors among adults living in the State of São Paulo, Brazil. J Am Diet Assoc. 2006; 106(12):2067-72.

17. Bowman SA, Lino M, Gerrior SA, Basiotis PP. The Healthy Eatins Index: 1994-96. Washington: U.S. Departament of Agrilculture; 1998. Publication $n^{\circ}$ CNPP-5.

18. World Health Organization. Obesity: preventing and managing the global epidemic. Geneva: WHO; 1997.

19. Sichieri R, Castro JFG, Moura AS. Fatores associado ao padrão de consumo alimentar da população brasileira urbana. Cad Saúde Pública. 2003;19(1): S47-S53.

20. Pereira RA, Andrade RG de, Sichieri R. Mudanças no consumo alimentar de mulheres do Município do Rio de Janeiro, Brasil, 1995-2005. Cad Saúde Pública. 2009; 25(11):2419-32.

21. Felippe F, Balestrin L, Silva FM, Scheneider AP. Qualidade da dieta de indivíduos expostos e não expostos a um Programa de Reeducação Alimentar. Rev Nutr. 2011; 24(6):833-44. doi: 10.1590/S14 15-52732011000600004.

22. Shah BS, Freeland-Graves JH, Facn RD, Cahill JM, Lu H, Graves GR. Diet quality as measured by the healthy eating index and the association with lipid profile in low-income women in early postpartum. J Am Diet Assoc. 2010; 110(1):274-79.

23. Garriguet D. Diet quality in Canada. Public Health Rep. 2009; 20(3):1-13

24. McCabe-Sellers BJ, Bowman S, Stuff JE, Champagne CM, Simpson PM, Bogle ML. Assessment of the diet quality of US adults in the 
Lower Mississippi Delta. Am J Clin Nutr. 2007; 86(1):697-706.

25. Nemby PK, Muller D, Hallfrisch J, Qiao N, Andres $\mathrm{R}$, Tucker KL. Dietary patterns and changes in body mass índex and waist circumference in adults. Am J Clin Nutr. 2003; 77(1):1417-25.

26. Suzuki CS, Moraes SA, Freitas ICM. Atividade física e fatores associados in adultos residentes in Ribeirão Preto, SP. Rev Saúde Pública, 2011; 45(2):311-20.

27. Alves A, Olinto MTA, Costa JSD, Bairros FS, Balbinotti MAA. Padrões alimentares de mulheres adultas residentes em área urbana no Sul do Brasil. Rev Saúde Pública. 2006; 40(1):865-73.

28. Fernandes LCL, Bertoldi AD, Barros AJD. Utilização dos serviços de saúde pela população coberta pela estratégia de saúde da família. Rev Saúde Pública, 2009; 43(4):595-603.

29. Freedman LS, Guenther PM, Krebs-Smith SM, Kott PS. A population's mean Healthy Eating Index-2005 scores are best estimated by the escore of the population ratio when one 24 -hour recall is available. J Nutr. 2008; 138(9):1725-29.

30. Freitas BSA, Carvalho CMRG. Relação entre fatores dietéticos, clínico-reprodutivos e estilo de vida em mulheres adultas e idosas com a osteoporose em Teresina - Piauí. Nutrire. 2008; 33(1):1-19.

Recebido em: 29/9/2011

Versão final em: 24/8/2012

Aprovado em: 5/9/2012 
